\title{
GENDER-SPECIFIC TREATMENT FOR FEMALE YOUNG OFFENDERS
}

Eureka

Volume 3, Number 1 (2012)

Lily Le

Psychology Intern, 2010 - 2011, Turningpoint Program, Alberta Hospital Edmonton

The rate of female youth being charged with serious violent crimes in Canada has more than doubled since 1986, rising from 60 per 100, 000 to 132 per 100, 000 by 2005 (Kong \& AuCoin, 2008). These numbers are much lower in comparison to male young offenders, but the increasing number of female youth entering the juvenile justice system calls for increased attention. Because the juvenile justice system has consisted of mostly males, the system is geared towards males and the specific needs of female young offenders have been overlooked.

Researchers have argued that although both sexes may commit the same violent crimes, such as murder and assault, they are gender-specific in their development and are therefore gender-specific in their pathways to offending as well (Belknap \& Holsinger, 1998). Nonetheless, male delinquency is seen as a behavioral issue that is dealt with through rehabilitation programs that focuses on behavior modification, whereas female delinquency is pathologized and seen as a psychiatric problem (Hartwig \& Myers, 2003). Thus, many young female offenders do not receive the gender-specific programs that they need. Moreover, the treatment literature on female young offenders is scarce in comparison to male young offenders.

Although there has been heightened interest in the idea of gender-specific treatment groups for incarcerated female youth, few programs have been implemented in Canada. Many of the groups for female young offenders in the United States concentrate on specific issues such as substance abuse, not a wide range of topics that this population are affected by and/or focused on preventing delinquent behavior in non-offending girls. Thus, research on the specific needs of female young offenders is required to inform treatment that targets the unique needs of this booming population. Informing a gender-specific programming begins with an increased understanding of how female young offenders differ from male young offenders in essential areas such as their trauma exposure, psychopathology, potential pathways to delinquency, and gender constructs.

\section{Trauma}

Female young offenders have reported experiencing a significant amount of trauma in their lives. Ariga et al's (2007) study on trauma exposure indicated that $76.5 \%$ of the delinquent female adolescents had experienced a traumatic experience, with sexual abuse being the most frequently reported (54.7\%). High reports of sexual abuse among young female offenders have been indicated in many other studies on this population. Siegal and Williams (2003) compared delinquency in girls who were sexually abused as a child to a non-abused group. The results indicated that a larger proportion of arrests were made for female delinquents who had a history of child sexual abuse in comparison to the match group in every category of crime examined (i.e. violent offenses, property offenses). Female delinquents who had a history of sexual abuse were 2.1 times more likely to be arrested than the comparison group. When the rate of offending in both groups was compared in adulthood, females with a history of child sexual abuse were twice as likely to be arrested as adults and the number of arrests was two times greater for a violent offense than when compared with the non-abused group. Although there are young male offenders that report being sexually abused as children, the number of female young offenders with this history is much more significant (Gover, 2004). For example, Alemagno, Shaffer-King \& Hammel's (2006) found that the female young offender participants reported unwanted sexual contact 15 times more than the male young offender participants. Female young offenders have also reported a greater incidence of physical and emotional abuse, along with physical neglect in comparison to male offenders (McCabe, Lansing, Garland and Hough, 2002). Many female young offenders experience violence within their own homes. In one study, families of female young offenders demonstrated high rates of domestic violence and physical abuse (Dixon, Howie, and Starling, 2004). Another study found that female young offenders, in comparison to their male counterparts, reported more physical violence in the home, less family support, and a fear of staying in the home (Alemagno, Shaffer-King, Hammel, 2006).

Trauma is not limited to interpersonal violence, but also includes witnessing violence, being confronted by bad news, and childhood maltreatment. Dixon et al (2005) found that $70 \%$ of the participants in their study reported witnessing a violent crime and $66 \%$ reported being confronted with traumatic news and in another study, $32.8 \%$ of the female young offenders reported being confronted with traumatic news (Ariga et al., 2007). These studies showed that the young female offenders often experienced multiple traumatic experiences.

Hence, the literature on trauma and offending show that females in trouble with the law have experienced victimization at much higher rates than the general population. Young female offenders typically experience more trauma than young male offenders, and are also more likely to be arrested than young male offenders, suggesting that their traumatic history may play a role in their delinquent behavior.

\section{Psychopathology}

Traumatic experiences such as experiencing and witnessing violence often leave devastating psychological repercussions, and since many offenders have experienced trauma, this may increase their risk of having psychological disorders. In comparison to non-offending adolescents, Dixon, Howie \& Starling (2004) found that youth in trouble 
with the law experience more psychological symptoms. Female young offender participants reported significantly more types of traumatic experiences than non-offenders, such as higher levels of personal victimization and exposure to violence. They found that female juvenile offenders report significantly more psychopathology than nonoffenders, with conduct disorder (CD) (91\%), substance abuse/dependence (85\%), alcohol abuse/dependence (56\%), depression (55\%) and post-traumatic stress disorder (PTSD) (37\%) being the most common disorders. Nearly half of the participants in this study also reported attempted suicide. All but one of the females had at least one psychiatric diagnosis and $78 \%$ met the diagnostic criteria for three or more diagnosis.

Specifically, sexual abuse among females is associated with higher rates of psychopathology, especially depression. Gover (2004) examined depression in adolescents who were incarcerated and had a history of child sexual abuse. Both males and females who have been sexually abused exhibited significantly higher levels of depression in comparison to boys and girls who have not been sexually abused. Female survivors of sexual abuse were more likely to experience depression and had the highest levels of depression when compared male survivors. The lowest levels of depression were found in boys who were not sexually abused.

PTSD has also been examined more readily in female juvenile offenders with a history of sexual abuse. Because sexual abuse occurs in much higher rates in females than males, many females with a history of sexual abuse experience PTSD in comparison to males (Ariga et al., 2007). McCabe et al. (2002) found that female delinquents have higher rates of parent reported and self reported psychological symptoms as well as DSM disorders when compared with male delinquents.

Dixon, Howie, Starling and Franzcp (2005) found that among the female young offenders in their study, the largest precipitator of PTSD was sexual abuse (70\%), despite other more frequently reported traumas such as witnessing a violent crime and being confronted with traumatic news. Females who experienced PTSD were more likely to report comorbid diagnosis such as depression, eating disorders, anxiety disorders, psychosis, substance/alcohol abuse and attempted suicide as opposed to males who are more likely to display comorbid attention-deficit/hyperactivity disorder (ADHD) disorder and CD.

Substance and alcohol abuse/dependence are also frequent diagnoses within the young offender population. There is a strong link between substance abuse and criminal activity among both male and female young offenders (Chassin, 2008). In one study, $85 \%$ of female young offenders reported having substance abuse/dependence, and $56 \%$ had alcohol abuse/dependence. Many of these participants reported that they started using drugs to cope with the flashbacks and nightmares associated with their PTSD (Dixon, Howie \& Starling, 2004).

\section{Potential Pathways to Offending}

There have been some ideas as to why the rate of female delinquency has increased over the years. One potential pathway to delinquency may be the due to the victimization of prior abuse and violence that occurred in female's lives as has been discussed earlier in this paper. Being victimized in childhood is a stronger predictor of offending for females than males (Cauffman, 2008).

Feiring, Miller-Johnson and Cleland (2007) found that stigmatization, such as self-blame, abuse-specific shame, and internalizing symptoms, such as PTSD and depression in sexual abuse survivors were related to anger and delinquent behaviors.

Schaffner (2002) suggested that sexual and physical violence against girls has provoked many girls to act out violently themselves. Her study examined the relationship between young female offenders and the violence experienced and witnessed in childhood. Over half of the participants in this study reported witnessing physical, sexual and emotional abuse of others. Many of them had witnessed the abuse of others on numerous occasions. However, very few defined these events as acts of violence or abuse, although a relationship was found between witnessing violence and later offending. The increased violent acts among young female offenders can be attributed to the normalization of witnessing others act out aggressively, often within their hostile home environment. Essentially, it may have become engrained in the females to act out violently in response to anger, as that has become a routine reaction which they have observed throughout their lives.

The increased violent crime by young female offenders could also be their way of fighting back in response to their victimization (Wolfe \& Tucker, 1998). Coping mechanisms that are seen at higher rates in females, like self-harm, provide a sense of control, however, acting out violently towards others may be an alternative method to regain some power. Morton and Leslie (2005) found that female young offenders who were victimized felt the need to regain the power and control that was taken away from them through their abuse experiences. The researchers interviewed clinicians who led therapeutic groups for female young offenders, many of which had a history of sexual abuse. The clinicians expressed that the girls' engagement in delinquent behaviors could be their way of regaining control and power in their lives after feeling powerless in their sexual abuse. The girls' they worked with attempted to gain power and control by putting their needs first, only doing something for others when it would benefit them, and by acting aggressively and manipulatively. As the clinicians pointed out, their methods of gaining power and control would ironically result in incarceration, where power and control were taken from them once again.

Prior victimization among young female offenders may not only be related to an increase in violent offending, but non-violent offending as well. In the United States, many female delinquents are charged with status offenses, (crimes that pertain to specific group of people, in this case, minors) such as running away, many of them fleeing from an abusive environment. Often, females who run away end up living on the streets, where they engage in theft, prostitution, and drug-related activities to survive (Wormer, 2010). Coinciding with idea that female violent offending 
could be used as a way to regain power, these previous non-violent activities may serve the same purpose. In one study, female sex workers with a history of sexual abuse reported that by exchanging sex for money, they felt a sense of control over their bodies (Campbell, Ahrens, Sefl, Clark, 2003).

Females often use drugs and alcohol as a coping mechanism for their trauma and psychopathology and therefore may have to continue to engage in high risk behaviors to fuel their substance abuse. Substance abuse has been shown to play a large role in the increase of female offending (Byrne \& Howells, 2002).

Unfortunately, these methods of survival could put females at greater risk for victimization. For example, a female prostitute with a substance abuse problem may increase her chances of being exposed to more traumas, such as sexual violence. As indicated earlier, many female young offenders who experience trauma often experience psychopathology (or mental health issues) such as PTSD and depression. Unfortunately, the unhealthy coping mechanisms commonly adopted by this group include substance abuse and acting out aggressively to themselves and/or others. This pattern of repeated victimization, psychopathology, and unhealthy coping strategies create a cycle of crime that is difficult to break. Thus, when compared with their male counterparts, female young offenders' pathways to offending and coping mechanisms are different and call for increased attention to the gender constructs that make up these differences.

\section{Gender Constructs and its Influence on Delinquent Behavior}

There is a desperate need for gender-specific programming for at risk youth because males and females develop differently and are taught to act in certain ways according to their gender. They also seem to be affected differently by similar histories. Sex is biologically determined, whereas gender is socially constructed by society. How males and females are gendered in society can help explain the gender specific trauma responses, psychopathology and potential pathways to female offending. In female young offenders who have experienced abuse by males, we can examine society's depiction of masculinity and femininity.

As children, males are often taught to be in control and aggressive and are therefore more prone to act out physically, whereas girls are taught to be submissive and polite and are therefore more likely to internalize their anger. Thus, it is not surprising that when it comes to the need to regain a sense of power, males typically exhibit physical and sexual violence and females are typically depressed and acting out through self-harm or suicide (Covington, 2003). These ideas of the dominant male and passive female are introduced to children as soon as they enter the world. Rough play among boys is tolerated and normal, but is usually not acceptable for girls. Specific toys teach children what type of play is expected of them; boys are usually involved in competitive and aggressive play with cars and actions figures, whereas girls are being prepared for their expected roles of nurturing mother and wife, tending to dolls and using Easy-Bake ovens. A large majority of fairy tales teach boys that they are to be brave and powerful, winning over princesses by slaying evil creatures, whereas girls are taught that happily-ever-afters are achieved when they, the passive in-need-of-rescuing females, are saved by the mighty Prince Charming. Aggression in males is often encouraged and condoned, allowing a culture where physical and sexual violence against females is normal. Females are often taught to be passive and are therefore often the victims of male's violent behavior, especially in sexual violence as indicated earlier.

These ideas that males are to be dominant and powerful, whereas females are to be their subordinates continue to be taught and reinforced in adolescence and adulthood. We are bombarded with these messages in the media; just watch a mainstream rap video and more often than not, there are males surrounded by money, cars and scantily clad women. Studies have illustrated that watching television has an impact on how we view gender roles (Lecroy and Daley, 2001). The ideal nuclear family still consists of the working man who is financially dependent, and the housewife who tends for the children. We live in a "rape-prone" culture, a culture characterized by high rates of rape. Our culture promotes sexual aggression in males and female objectification is common. Examples of this include mainstream pornography which has become increasingly degrading and violent against women, yet is a multi-billion dollar business: $\$ 10$ billion in the U.S. and $\$ 57$ billion worldwide annually (Dunlap, n.d.). In a Canadian survey, $87 \%$ of women reported experiencing sexual harassment ("Measuring Violence Against Women, 2006). Sexual assault occurs in 1/3 females worldwide (The World Bank, 2001). Intimate partner abuse and domestic violence (survivors being mainly females) rank at the top of the list in the number of calls to the police. These ideas not only make it inviting and normal for males to victimize females, but it can convince some females that being victimized by males is a normal part of their gender. Some females, especially those who are victimized repeatedly, do not see abuse to them as wrong, but accept that this is their place in society.

Moreover, young females' sense of identity becomes defined by society's messages of femaleness, coupled with how they are constantly subordinate to their male counterparts. The quest to determining one's self-identity is often already difficult for young females, not to mention female young offenders who attempt to do this while incarcerated. For delinquent females, this sense of identity is often related to being a source of pleasure for males, an identity that would increase risky sexual behaviors and criminal activity. Morton and Leslie's (2005) study illustrates that cultured norms of the passive and receptive sexual female are evident in their sense of self. The definition of sexual activity was unclear to many of the young female offenders in the study, most of whom were sexual abuse survivors. However, the idea that women are sexual objects was evident. Many of these girls reported that their ultimate goal was to be with a partner who loved them. Unfortunately, many of their male partners were abusive and encouraged them to participate in delinquent acts. 
Another study found that female young offenders had difficulty defining themselves as females or individuals, instead basing their sense of self on others, usually their male partners. Moreover, they had poor self-esteem, poor female role models, and a poor view of womanhood. More female participants reported exchanging sex for money and drugs, as well as having a sexually-transmitted infection (STI) in the past six months when compared to the male participants. More than half of the male and female participants reported engaging in sexual intercourse before the age of 13 , a risk factor itself for offending. $62.1 \%$ reported engaging in sexual intercourse without a condom (Alemagno, Schaffer-King and Hammel, 2006).

The ways in which females display their aggression is also gendered. Physical aggression is more typical in boys, whereas relational aggression has been observed more in girls (Crick \& Rose, 2000). Relational aggression can be defined as a form of aggression in which harm is caused to another by threatening or damaging their relationships. Because it goes against conventional gender norms for females to act out violently, they often use relational aggression to hurt others, usually other females. This form of aggression has also been observed to get more sophisticated and complex as children mature into adolescence, including both direct and indirect methods of harming relationships (Crick, Casas, and Nelson, 2002). Although physical aggression amongst females is not as common as male physical aggression, the rate in violent offending amongst female adolescents has ignited, and thus, both physical and relational aggression are deserving of discussion with this population.

Gender constructs shape who girls are and who they will become. Gender roles define girls' responsibilities and rightful place in society. Gender stereotypes constrict girls to how they should think and behave. Hence, genderspecific treatment is needed for young female offenders to explore, critique, re-establish, or even develop their roles and identities as empowered females who are capable of change and success.

\section{Case Illustrations of Gender-specific Programs}

There have been few gender-specific programs that have been implemented for young female offenders in a correctional or treatment facility. In the United States, more steps have been taken in this direction of gender specific programs, especially after the 1992 Reauthorization of Juvenile Justice and Delinquency Prevention Act (JJDP Act) which included the component of gender specific services. ("The Juvenile Justice and Delinquency Prevention Act of 1974," n.d.) Zahn et al. found sixty-two programs aimed at delinquent girls and girls in the juvenile justice system in the United States. Out of these programs, nine were targeted towards young female offenders and had at least one evaluation conducted of the program. Although some of these gender-specific programs are prevention programs and/or conducted in places other than the correctional setting, they revolve around and discuss similar models and themes that may be applicable to young female offenders. The programs listed below focus on gender specific issues for at risk youth in a variety of settings, and have been found to produce significant changes in the lives of many female adolescents.

One popular program, Girls' Circle has trained more than 500 organizations and has been implemented in schools, clinics, agencies, homes and group homes. Girls' Circle is a strengths-based group utilizing a model that combines relational theory, resiliency practices, and skills training with the goal of enhancing courage and growth in the group. Gender specific topics include being a girl, friendship, body image, diversity and identity ("How It Works," 2011). In 2007, Roa, Irvine, and Cervantez conducted a study on the effectiveness of Girls' Circle and found that the group improved girls' well-being. Short-term effects include feeling good about their body and expressing verbal affection to others, whereas long-term effects included a decrease in self-harm and alcohol use and an increase in self-efficacy. However, girls' held in juvenile detention, residential facility or other secure facilities did not display as much as an improvement when compared to other girls' who were not in these settings. Gains in short-term skills and self-efficacy of girls in a secure setting were lower than girls who were not. As suggested by the researchers, girls in detention may have specific needs in regards to these short-term skills and self-efficacy and further steps need to be taken to understand the experiences of this specific population. Overall, Girls' Circle has shown to be beneficial to many young females.

In North Carolina, the Holistic Enrichment for At-Risk Teens (HEART) program has been designed to reduce recidivism and substance abuse relapse among young female offenders. The program uses gender-specific perspectives, such as feminist and relational theories along with the Multilevel Risk Model which takes into account the multiple levels of risks (biological, psychological, developmental, familial/communal and societal) that play a role in female delinquency. The program uses the BioPsycho-Social-Spiritual (BPSS) model of addiction which states that many factors, such as biological, psychological, social, spiritual, and developmental, may play a role in substance abuse/dependence. Therapy and groups cover topics such as female socialization, gangs, relational aggression, and sisterhood (Welch, Roberts-Lewis, and Parker, 2009). Girls in the HEART program improved more in their use of social support, perceived support of friend, and peer acceptance when compared with the non-treatment group. Girls in the HEART program also experienced improved self esteem, social development family relationships, and educational status in relation to the comparison group. Also, school engagement and grades improved in the treatment group (Kirk and Griffith, 2004, as cited in Zahn, 2009).

A state-wide day treatment program program in Florida called PACE (Practical Academic Cultural Education) Center for Girls, Inc. is a prevention program provided for girls who may be involved in risky activities or crime. The program recognizes the relationship between prior victimization and crime and therefore provides a gender responsive approach to education, counseling and career planning. The purpose of the program is to prevent female delinquency and risky behaviors by strengthening educational and vocational 
areas in the girls' lives ("Our Goals," 2010). A 2009/2010 evaluation of the program indicated that for girls who were transitioning in 2007-2008, 93\% were not adjudicated nor had adjudication withheld while they were enrolled in the program, 94\% were not adjudicated nor had adjudication withheld within 6 months from the day program, and 91\% were not adjudicated nor had adjudication withheld within 1 year from the day program. Academic goals, such as having at least $25 \%$ of the girls mainstream back into a public school and having at least $75 \%$ of the girls increase their academic functioning (i.e. GPA, specific subject assessments, earning credits and completing courses) were all exceeded with $64 \%$ and $95 \%$ of girls meeting both goals, respectively. Altogether, 21,000 girls have been served across the 17 PACE centers and programs in Florida. PACE's focus on girls' strengths and protective factors have illustrated that participants who complete the program are able to make more positive choices, such as concentrating on educational and vocational goals, which prevent delinquency and high risk behaviors.

Lastly, the Go Grrrls Program is an after school prevention program for middle-school girls that aims to foster healthy gender role identification and healthy psychosocial development in today's society. The program focuses on gender specific issues such as body image, self-esteem, friendship, sex, success, and independence. Lecroy (2004) conducted an evaluation of the program. Participants were divided in to a treatment group who attended the program, and a control group who did not attend the program. Before and after each intervention, participants filled out self-questionnaires with 8 different measures such as body image, self efficacy, and assertiveness. The evaluation found that for variables such self-efficacy and self-liking and competence, girls in the program displayed a significant positive change in comparison to the control group. Moreover, a significant between group effect was found, with the treatment group reporting a greater increase in body image, assertiveness, attractiveness, and self liking and competence than the control group.

With little gender specific programs for incarcerated female delinquents, there is a scarcity of literature on the evaluations of these programs. There is research to show that gender specific treatment does work, especially when the programs cover multiple aspects of the girls' lives (Cauffman, 2008). Although gender-specific treatment has indicated that although gender-specific programming does have positive effects in areas such as education, relationships, self-esteem, self-efficacy and other socialpsychological outcomes like body image, there is little evidence to illustrate that this type of programming reduces recidivism, despite improving girls' quality of life. This may suggest that these programs work while girls remain in the program (Zahn et al., 2009). Covington (2000) stressed the importance of creating a safe space through site and staff selection, program development, content and material that depicts the truths of the lives of girls and women in our society.

There have been implications from experts in the field on what key elements are required to facilitate successful gender-responsive programming for females. It has been suggested that because female delinquents often use anger as a mode of survival, programs should begin with topics such as self-esteem building, empathy training and maintaining healthy boundaries to build strength within them and therefore be less likely to resort to anger to gain power (Morton \& Leslie, 2005). Garcia and Lane (2009) found that a common desire amongst young female offenders in their girls' group was the desire to have a voice and play an active role in decision-making regarding their care. These girls longed for staff to show them that they cared. These considerations, along with the successful elements of case illustrations mentioned should be taken into account and form a basis for future gender specific programs for young female offenders.

\section{The Turningpoint Program}

The Turningpoint program at Alberta Hospital is an 18-bed facility that strives to provide the best possible inpatient assessment, treatment, and rehabilitation for youth in trouble with the law. This mission is accomplished by utilizing best practices in treatment and sparking new research. Although there has been an increase in the number of female young offenders admitted for treatment and assessments over the years, there has been no treatment group that addresses the needs of girls specifically. As supported by the literature, the young female offenders who stay at the Turningpoint program are violent offenders who often have experienced trauma and have been diagnosed with a number of psychopathologies. Many come from backgrounds where they have witnessed and experienced violence. Many have had a history of repeated victimization. Most commonly, these girls share the similar experience of being a young woman in trouble with the law, and thus share a number of gendered experiences. With the majority of the young offenders on the unit being male, these girls do not have a space where they can come together to discuss gender specific issues. Thus, forming a gender specific group for the female young offenders will provide the opportunity for these girls to have a safe environment where their experiences can be acknowledged, where they can learn about their gender specific issues, and where they can be empowered to make positive life changes.

\section{The Turningpoint Girls' Group}

Similar to the case illustrations discussed, the Turningpoint Girls' Group will use strengths-based, empowerment approach and focus on a variety of topics from a female perspective. Like the PACE Center for Girls, Inc., the group will emphasize the relationship between childhood abuses and delinquency ("Our Goals," 2010). Thus, the group is rooted in the feminist pathways perspective, which suggests that delinquent behaviors may derive from a number of areas that exist within our patriarchal society, such as learned gender roles, structural oppression, female's victimization by males, and female's responses to male power. This theory posits that patriarchy plays a large role in delinquency and therefore, both boys' and girls' potential pathways to delinquency may be gender specific (Belknap \& Holsinger, 2006). The theory is not limited to just gender, but other intersections such as 
race and class.

Belknap \& Holsinger (2006) determine how feminist pathways theory contributes to better understanding the gendered risks associated with delinquency. For measures of abuse, girls reported significantly more abuse than boys for almost every variable. For measures of family, girls had the worst home experiences, reporting significantly more abandonment than boys, and reporting that someone else had helped raise them aside from their parents more than boys. In terms of mental health, girls reported significantly higher likelihood of mental health problems. For measures of school experiences, girls were significantly more likely than boys to drop out of school or quit. Consistent with the literature presented on gender specific issues relating to female delinquents, the feminist pathways theory provides the most support for determining not only girls' risks, but boys' risks as well. Feminist pathways theory has illustrated the importance of understanding how trauma is a risk factor for delinquency, and thus needs to be incorporated into other theories that do not address this issue when working with delinquents. The majority of gender-specific programs examined in this study targets similar risk and protective factors, and a few others target specific risk factors such as substance abuse. However, very few target abuse and neglect, family and peer relationships, mental health and gang involvement although these risk factors are extremely influential in the pathway to delinquency. All in all, the findings of girls' experiences are most consistent with feminist pathways theory and thus life events of delinquent females, such as traumatic experiences, must be considered for effective gender specific programming.

In terms of counseling paradigms, an empowerment/ strengths-based approach will be taken in the Turningpoint Girls' Group. Cognitive-behavioral therapy has served as the dominant paradigm in corrections. This approach focuses on changing offenders' thinking patterns, offenders' pathology, and preventing recidivism. The strengths-based approach, on the other hand, focuses on multiple interactive levels of influence, the person as a whole, and the offenders' sense of motivation to change and well being. This approach is rooted in the belief of human potential (Wormer, 2010).

The group will be available for all female-identified patients on the unit, both treatment patients (those who are admitted to the treatment program) and remand patients (those who are not admitted to the treatment program, but awaiting assessment). The group will be psychoeducational, presenting information and ideas through education and activities regarding a variety of genderspecific issues in which the girls can relate with. Similar to previous groups that have been conducted with this population, the girls' group will cover topics such as selfesteem, self-identity, anger, healthy relationships (familial, peer, and romantic), sexuality, risk and protective factors, gender specific trauma, and girls in relation to the world. Education and discussion of these issues will hopefully promote growth, healing and empowerment for these girls. Discussion of the issues will be encouraged and done so in a safe and supportive environment. The group will always take place in a quiet room off the unit to instill consistency, safety and comfort. Weekly sessions of 1-1.5 hours will take place over a course of 8-12 sessions. Pre and post self-evaluations measuring concepts such as self-esteem, knowledge, and overall satisfaction of the group will be handed out to all the patients. Homework will be given and collected on a weekly basis.

The group will be facilitated by the author, along with another female co-facilitator. These facilitators will be aware of the girls' traumatic experiences, offense(s), current mental health, and needs. Facilitators will work with the feminist pathways theory in mind, and using an empowerment approach to foster a sense of confidence within the group. It is key that facilitators develop good rapport with the girls, be sensitive to their history and current mental state, and work to foster an environment of trust.

The author is hopeful that gender-specific treatment for the young female offenders of the Turningpoint program will be helpful in promoting positive change in the girls' lives. Perhaps with the implementation of this program over time, gender specific programming for this population will be of greater interest in Canada and the specific needs of delinquent females will be less likely to be overlooked.

\section{References}

Alemangno, S., Shaffer-King, E., \& Hammel, R.(2006). Juveniles in detention: How do girls differ from boys? Journal of Correctional Health Care, 12(1), 45-53.

Ariga, M., Uehara, T., Takeuchi, K., Ishige, Y., Nakano, R., \& Mikuni, M. (2007). Trauma exposure and posttraumatic stress disorder in delinquent female adolescents. The Journal of Child Psychology and Psychiatry, 49(1), 79-87.

Belknap, J., \& Holsinger, K. (1998). An overview of delinquent girls: How theory and practice failed and the need for innovative changes. In R. T. Zaplin (Ed.), Female Offenders: Critical Perspectives and Effective Interventions, Gaithersburg, MD: Aspen Publishers.

Belknap, J., \& Holsinger, K. (2006). The gendered nature of risk factors for delinquency. Feminist Criminology, 1(1), 48-71.

Byrne, M., \& Howells, K. (2002). The psychological needs of women prisoners: Implications for rehabilitation and management. Psychiatry, Psychology and Law, 9(1), 34-43.

Campbell, R., Ahrens, C., Sefl, T., \& Clark, M. (2003). The relationship between adult assault and prostitution: An exploratory analysis. Violence and Victims, 18(3), 299-317.

Cauffman, E. (2008). Understanding the female offender. The Future of Children, 18(2), 119-134.

Chassin, L. (2008). Juvenile justice and substance use. The Future of Children, 18(2), 165-183.

Covington, S. (2000). Creating gender-responsive programs: The next step for women's services. Corrections Today, 63(1), 85-87.

Covington, S. (2003). A woman's journey home: Challenges for female offenders. In J. Travis \& M. Waul (Eds.), Prisoners once removed: The impact of incarceration and reentry on children, families, and communities. Washington, DC: Urban Institute Press.

Crick, R., \& Rose, A. (2000). Toward a gender-balanced approach to the study of socio emotional development: A look at relational aggression. In Miller, P., \& Scholnick, E. Toward a Feminist Developmental Psychology. New York, NY: Routledge.

Crick, R., Casas, F., \& Nelson, A. (2002). Toward a more comprehensive understanding of peer maltreatment: Studies of relational victimization. Current Directions in Psychological Science, 11(3), 98-101.

Dixon, A., Howie, P., \& Starling, J. (2004). Psychopathology in female juvenile offenders. Journal of Child Psychology and Psychiatry, 45(6), 1150-1158. 
Dixon, A., Howie, P., Starling, J., \& Franzcp, J. (2005). Trauma exposure, posttraumatic stress, and psychiatric comorbidity in female juvenile offenders. American Academy of Child and Adolescent Psychiatry, 44(8), 798-806.

Dunlap, B. (n.d.) Porn, meth and violence: Making some connections. Crisis Connection. Retrieved from http://www.crisisconnectioninc. org/pdf/Porn.pdf

Feiring, C., Miller-Johnson, S., \& Cleland, C. (2007). Potential pathways from stigmatization and internalizing symptoms to delinquency in sexually abused youth. Child Maltreatment, 12(3), 220-232.

Garcia, C., \& Lane, J. (2009) What a girl wants, what a girl needs: Findings from a gender-specific focus group study. Crime \& Delinquency, published online April 3, 2009. Retrieved from http:// cad.sagepub.com/content/early/2009/04/03/001112

Gover, A. (2004). Childhood sexual abuse, gender, and depression among incarcerated youth. Internation Journal of Offender Therapy and Comparative Criminology, 48(6), 683-696.

Hartwig, H., \& Myers, J. (2003). A different approach: Applying a wellness paradigm to adolescent female delinquents and offenders. Journal of Mental Health Counselling, 25(1), 57-75.

How It Works. (2011). Girls Circle. Retrieved from http://www.girlscircle. com/ how it works.aspx

Kirk, R., \& Griffith, D. (2004). End-of-year report to the Department of Juvenile Justice and Delinquency Prevention for state fiscal year 2004: Evaluation summary of assessment information for residents of the HEART and BEST programs at the Samarkand Youth Development Center. Chapel Hill: University of North Carolina, Chapel Hill, Jordan Institute for Families, School of Social Work. Cited by Zahn, M., \& Day, J. (2009). Determining what works for girls in the juvenile justice system: A summary of evaluation evidence. Crime \& Delinquency, 55(2), 266-293.

Kong, R., \& AuCoin, K. (2008). Female offenders in Canada. Juristat: Canadian Centre for Justice Statistics, Statistics Canada, Catalogue 85-112-XIE, 28(1).

Lecroy, C. (2004) Experimental evaluation of "Go Grrrls" preventive intervention for early adolescent girls. The Journal of Primary Prevention, 25(4), 457-473.

Marx, M. PACE outcome measure evaluation report (July 1, 2009 - June 30, 2010). PACE. Retrieved from http://www.pacecenter.org/images/ stories/PACE_Outcome_Measures_Report_0\%20-10.pdf
McCabe, K., Lansing, A., Garland, A., \& Hough, R. (2002). Gender differences in psychopathology, functional impairment, and familial risk factors among adjudicated delinquents. American Academy of Child and Adolescent Psychiatry, 41(7), 860-867.

Measuring Violence Against Women: Statistical Trends. (2006). Statistics Canada, Catalogue no. 85-570-XIE.

Morton, G., \& Lesley, L. (2005). The adolescent female delinquent: A feminist developmental analysis. Journal of Feminist Family Therapy, 17(1), 17-50.

Our Goals. (2010). PACE. Retrieved from http://www.pacecenter.org/

Roa, J., Irvine, A., \& Cervantez, K. (2007). Girls Circle national research project. Girls Circle. Retrieved from http://www.girlscircle.com/docs/ Final_Report_2007.pdf.

Schaffner, L. (2007). Violence against girls provokes girls' violence: From private injuryto public harm. Violence Against Women, 13(12)12291248.

Siegel, A., \& Williams, M. (2003). The relationship between child sexual abuse and female delinquency and crime: A prospective study. Journal of Research in Crime and Delinquency, 40(1), 71-97.

The Juvenile Justice and Delinquency Prevention Act of 1974: Prior Federal Juvenile Delinquency Activity. (n.d.). Retrieved from Office of Juvenile Justice and Delinquency Prevention, http://www.ojjdp.gov/ compliance/jidpchronology.pdf

The World Bank. (2001). Cited in Worldwide Sexual Assault Statistics. (2005). George Mason University Sexual Assault Services.

Welch, C., Roberts-Lewis, A., \& Parker, S. (2009). Incorporating gender specific approaches for incarcerated female adolescents: Multilevel Risk Model for practice. Journal of Offender Rehabilitation, 48(1), 67-83.

Wolfe, L., \& Tucker, J. (1998). Report of the Summit on Girls and Violence. Washington, DC: Center for Women Policy Studies.

Wormer, Katherine Van. (2010). Working with female offenders. Hoboken, New Jersey: John Wiley \& Songs, Inc.

Zahn, M., \& Day, J. (2009). Determining what works for girls in the juvenile justice system: A summary of evaluation evidence. Crime \& Delinquency, 55(2), 266-293. 